\title{
Design of WSN Based Intelligent Trash Bin Monitoring System
}

\author{
Qingmei LV 1 , a
}

\author{
1Department of Information and Electronic, Yuanpei College, Shaoxing University \\ 2799 Q un-xian Road Central, J ing-hu New District, Shaoxing, P.R. China 312000 \\ alv lucy@yeah.net
}

\section{Keywords: Intelligent Trash Bin; WS N; Solar Energy; Remote Monitoring}

\begin{abstract}
With the development of the society and the improvement of the living standard, people are to pursue more clean, healthy and harmonious living environment. And the trash management is one of the important factors. The aim of this paper is to design an intelligent trash bin monitoring system to improve the trash management. The system involves MCU controllers, solar panels, ultra-sonic sensors, wireless sensor network, GPRS, remote server and so on. The system can detect people who go closely to the trash bin and flip the lid automatically. It can also measure the trash level, send the level data via WSN and GPRS network to the server in the remote center, and store the data in a database. A web GUI interface can show the state of each trash bin, and remind the workers to deal with the trash in time. This system can enable more efficient management of the trash bins.
\end{abstract}

\section{Introduction}

Municipal solid waste (MSW) increases about 8\%-10\% per year[1] with the rapid development of the society. How to store and deal with MSW becomes an important factor which influences the good living environment. Especially in the locations with high traffic, such as communities and schools, trash bins are placed more intensive and the amount of waste is much larger. If not for timely cleanup, the waste piled up on the surrounding environment will cause serious pollution and environmental damage, and may even cause secondary pollution. Current treatment situation of trash bins in the region is that the sanitation workers inspect, clean them one by one in order to prevent a full trash bin. This requires a lot of manpower and material resources. The waste disposal cost is high. According to statistics, collection and transportation of MSW costs about $40 \%$ percent of total waste disposal costs[2]. In order to save resources, it's necessary to design an intelligent trash bin monitoring system which can acquire trash bin status information in real time[3, 4].

In China, there has been good researches on the MSW disposal flow. And some achievement of these researches are in use, such as MSW classified and transportation system of Pudong new area[5]. But for the intelligent trash bins which directly face to people, there has not been any good, large-scale business cases. However, the research on intelligent trash bins is always carried out. Zhou HJ[6] et al. proposed a smart bin based on RF and infrared technologies which can flip the lid automatically. Fan $\mathrm{LN}$ [7] et al. introduced a method to detect if the trash bin is full or not based on infrared sensing. Jin $\mathrm{ZM}[8]$ et al. presented a solution to compress and package the trash automatically. Xin ZH[9] et al. demonstrated how to measure the trash level with ultra-sonic sensor and transmit the data by ZigBee. Throughout these cases, most of them just realized intelligence for single trash bin. Some could transmit data, but only for short-distance communication. In order to construct a region monitoring system, we proposed an improved solution. Based on the single intelligent trash bin, we introduced solar energy for power supply, employed WSN (Wireless Sensor Network) technology based on ZigBee plus GPRS for long distance communication, and set up a remote monitoring platform based on $\mathrm{B} / \mathrm{S}$ architecture.

\section{System Architecture}

The architecture of the proposed intelligent trash bin monitoring system is illustrated in Fig.1. The proposed model consists of Trash Bins with intelligent controller and solar energy unit, wireless data communication and a remote monitoring center. The intelligent controller is responsible for monitoring 
trash bin's status, gathering the status data and sending them to the remoter data server by wireless communication technologies including ZigBee and GPRS. The solar energy unit supplies the power to the controller and other related modules. The server deals with the receiving data and stores them into the database, while a web GUI based interface gets the data from the database and shows the trash bin status to the users.

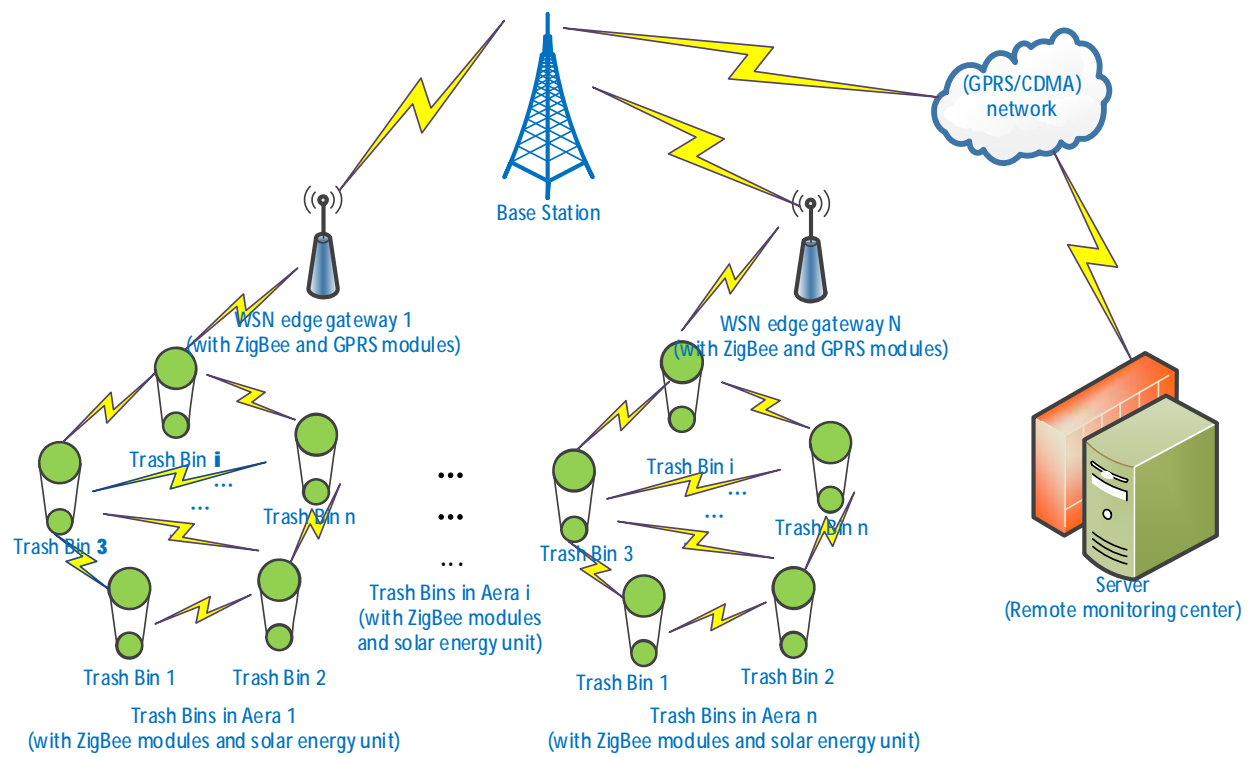

Fig. 1 Architecture of Intelligent Trash Bin Monitoring System

\section{System Implementation}

Trash bin state acquisition. In order to prevent pungent smell from the trash bin, usually there is a lid on the trash bin with close state. In normal situation, namely the bin is not full, when people walk closely enough(less than one meter) to the trash bin, the infrared sensor equipped on trash bin will detect him/her. And the controller will get a signal to trigger the motor to open the lid. If the trash bin is full or the power is lower than the preset value, the lid will not open. But meanwhile there is a led to indicate that the bin is not available.

For detecting the trash level, we introduced the ultra-sonic sensor to measure the trash bin capacity state. In normal, for each time the lid is open and close, the controller will check the trash level once, and store the level data into the rom. The controller will send the newest measured level data to the remote monitoring center via ZigBee and GPRS periodically, say 1 hour. But once the measured level is full, the controller will send the level data to the monitoring center immediately, and lock the lid, enable the led indicator meantime.

On the other hand, when the controller detects that trash bin is not dealt with in time, such as one day with full state or not full with three days, it will trigger an exception message, and send it to the monitoring center to remind the worker to check the trash bin in field. Fig. 2 hows the processing flow. Data transmission. ZigBee is a standards-based technology that addresses the unique requirements of most remote monitoring and control and sensory network applications[10]. It has features such as self-configuring, self-healing, low-cost, low-power-consumption. Here we use the ZigBee modules based on CC2530 to construct the subnet for transmitting the state data of trash bins to the edge gateway. Each ZigBee module in trash bin is not only a network node but also a router. That is to say it is a data transceiver as well as a repeater which can forward the data to the next node. When the controller is power on, it can measure the trash level of the bin, the left battery capacity and some error information. And then it can trigger the ZigBee module to send these data to the edge gateway, maybe via some other nodes. The edge gateway relay them to the remote monitoring center through the GPRS. The transmitting flow is shown in Fig. 3. 


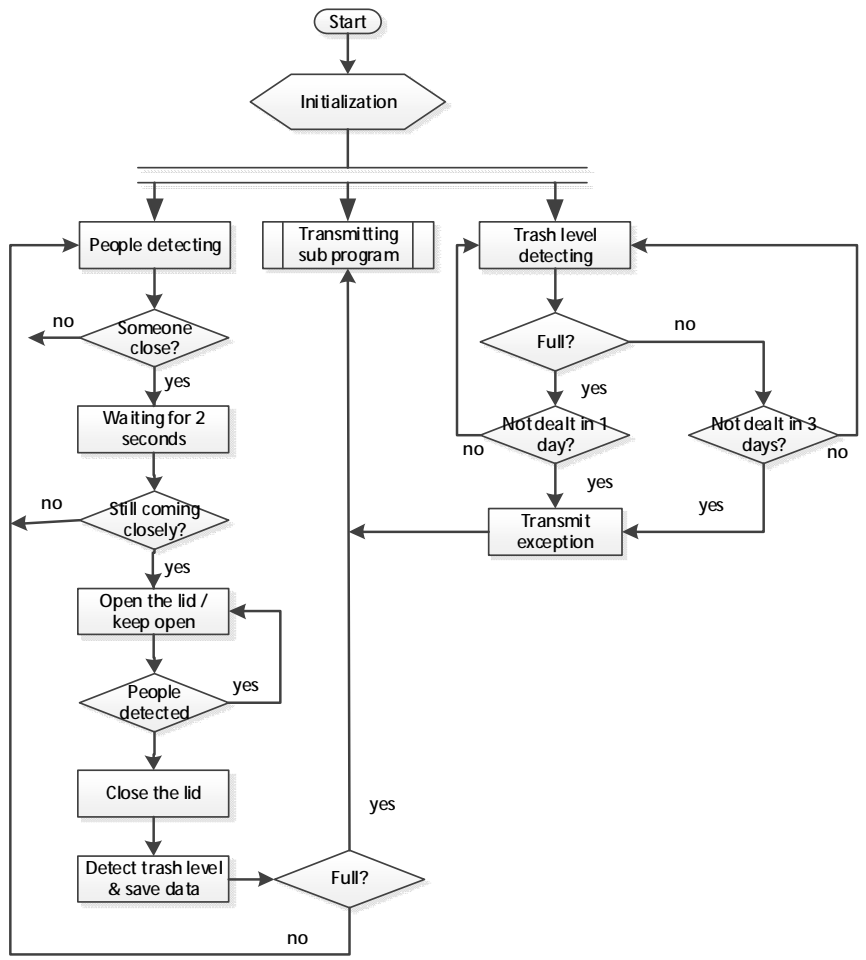

Fig. 2 controller processing flow chart

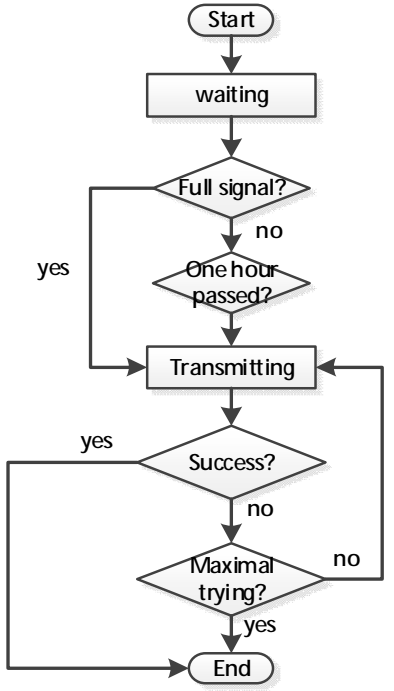

Fig. 3 transmitting flow chart

Solar Energy Unit. Solar energy unit is composed of a solar panel and a solar battery group. A controller is used to control the working process of solar energy unit. The controller will detect the battery's voltage level in order to decide whether it needs to be charged or not. And the controller will also measure the solar panel's parameters so that it can judge if the panel is ready to charge the battery. In the other hand, if the battery is in low voltage state, it will stop to supply the power to protect itself. Fig. 4 Shows the related circuit.

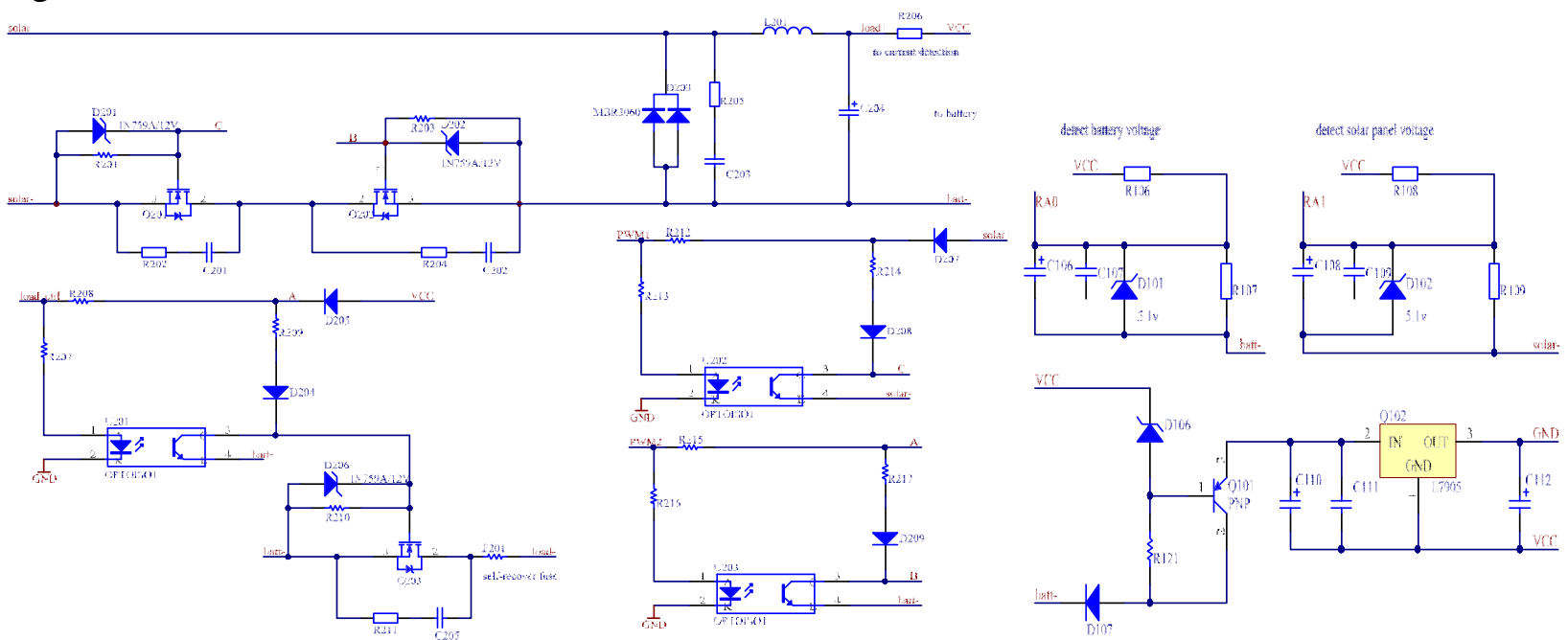

Fig. 4 main circuits of solar energy power supply including charging and discharging, voltage detection and power supply.

Remote Monitoring Center. The remote monitoring center is the junction for the trash bin state data from all edge gateways. The server in the center connects with the edge gateways by GPRS network, receives and stores the data, analyzes the data for further processing. We constructed a proper relational model and employed the SQL Server to store and manage the data. A web GUI based application is designed using HTML, C\#, JavaScript to represent the status of each trash bin, the immediately alert for the full trash bins, and the exception messages needed to be handled. Fig. 5 presents the web interface of the monitoring system. 


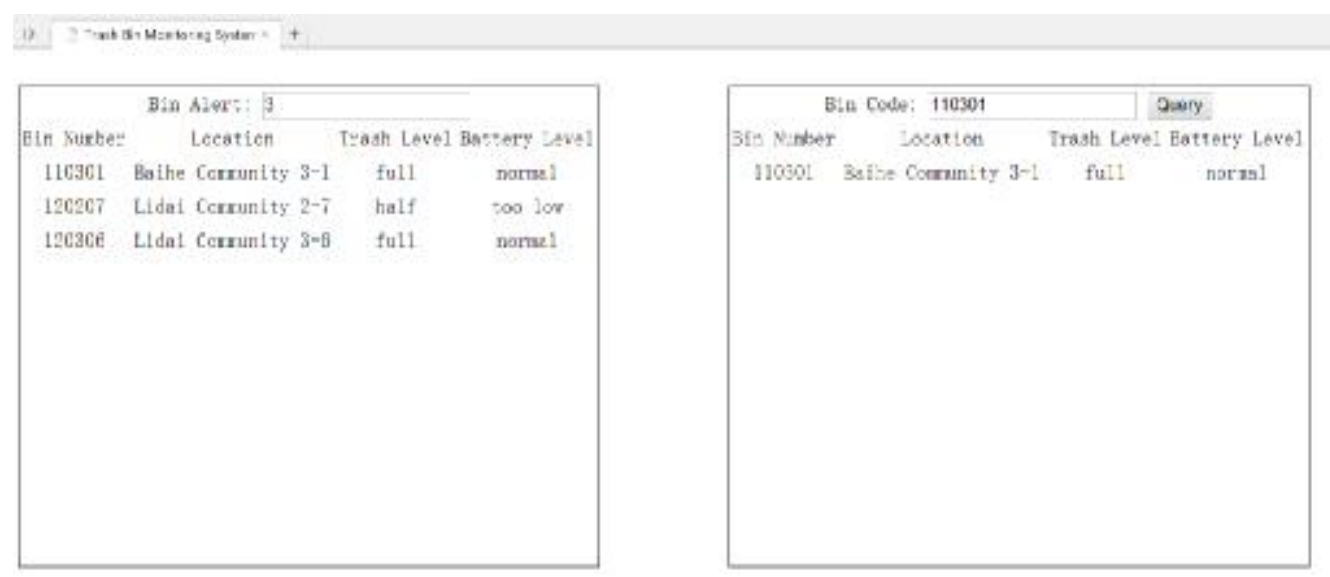

Fig. 5 web interface of the monitoring system

\section{Conclusion}

A WSN Based Intelligent Trash Bin Monitoring System is presented in this paper. It can detect the people who want to throw the rubbish, and can flip the lid automatically. It can monitor the trash level in real time, send the level data periodically to the remote center via ZigBee based WSN and GPRS, and save these data into the database. The electronic devices of the trash bin are all powered by the clean solar energy which is environment-friendly. And a simple web application is developed to show the trash bin level and alerts to remind workers to deal with the trashes in time. Moreover such a system can give a significant increase of convenience and cost effectiveness. However the trashes in the bin may be soft and loose, and can be compressed to extend the capacity of the trash bin. The intelligent trash bin will be more effective and better if it can equip with the compression device.

\section{Acknowledgements}

This work was financially supported by Zhejiang province education department scientific research project (Y201431501).

\section{References}

[1] Zhang YM, Shang XB, Li KM, et al. Technologies status and management strategies of municipal solid waste disposal in China. Ecology and Environmental Sciences, 2011. 20(2): P.389-396. (in Chinese)

[2] Song W, Liu JG and Nie YF. Study on Route Optimization for Municipal Domestic Waste Collection and Transportation. Environmental Sanitation Engineering, 2008. 16(1): p. 11-12, 15. (in Chinese)

[3] Hannan, M.A., et al. An automated solid waste bin level detection system using Gabor wavelet filters and multi-layer perception. Resources, Conservation and Recycling, 2013. 72: p. 33-42.

[4] Glouche, Y. and P. Couderc. A Smart Waste Management with Self-Describing objects. in The Second International Conference on Smart Systems, Devices and Technologies (SMART'13). 2013.

[5] Peng B. Applicating Pilot of Domestic Waste Classification Collection \& Transportation System and Informatization Supervision Technology in Pudong New Area. Environmental Sanitation Engineering, 2013. 21(1): p. 30-31, 34. (in Chinese)

[6] Zhou HJ and Xu JB. Design of a New Intelligent Garbage Can. Journal of Guangdong University of Technology, 2006. 23(3): p. 85-88. (in Chinese)

[7] Fan LN and Bai ZQ. The design of intelligent waste bin system based on single-chip microcomputer. EIC, 2006. 15(6): p. 16-17. (in Chinese)

[8] Jin ZM, Liu WT, Ni ZJ, et al. Design of Automatic Compression-Packaging Trash Bin. Journal of shanghai university of engineering science, 2010. 24(2): p. 110-113. (in Chinese)

[9] Xin ZH, Lu HM, Hu LY, et al. The design of intelligent garbage can system based on internet of things. EIC, 2011. 18(6): p. 37-39. (in Chinese)

[10] Hwang, S. and D. Yu, Remote Monitoring and Controlling System Based on ZigBee Networks. International Journal of Software Engineering and Its Applications, 2012. 6(3): p. 35-42. 